OPEN ACCESS

Edited by:

Jing-Ning Zhu,

Nanjing University, China

Reviewed by:

M. Gustavo Murer,

Universidad de Buenos Aires,

Argentina

Gordon William Arbuthnott, Okinawa Institute of Science and Technology Graduate University, Japan

*Correspondence: Steven M. Graves gravess@umn.edu

Received: 08 January 2019 Accepted: 28 February 2019 Published: 15 March 2019

Citation: Du Y and Graves SM (2019) Spiny Projection Neuron Dynamics in Toxin and Transgenic Models of

Parkinson's Disease. Front. Neural Circuits 13:17. doi: 10.3389/fncir.2019.00017

\section{Spiny Projection Neuron Dynamics in Toxin and Transgenic Models of Parkinson's Disease}

\author{
Yijuan Du and Steven M. Graves* \\ Department of Pharmacology, University of Minnesota, Minneapolis, MN, United States
}

Parkinson's disease (PD) is the most common neurodegenerative movement disorder that results from the progressive degeneration of substantia nigra pars compacta $(\mathrm{SNc})$ dopamine (DA) neurons. As a consequence of SNc degeneration, the striatum undergoes DA depletion causing the emergence of motor symptoms such as resting tremor, bradykinesia, postural instability and rigidity. The primary cell type in the striatum is the spiny projection neuron (SPN), which can be divided into two subpopulations, the direct and indirect pathway; the direct pathway innervates the substantia nigra pars reticulata and internal segment of the globus pallidus whereas the indirect pathway innervates the external segment of the globus pallidus. Proper control of movement requires a delicate balance between the two pathways; in PD dysfunction occurs in both cell types and impairments in synaptic plasticity are found in transgenic and toxin rodent models of PD. However, it is difficult to ascertain how the striatum adapts during different stages of PD, particularly during premotor stages. In the natural evolution of $\mathrm{PD}$, patients experience years of degeneration before motor symptoms arise. To model premotor PD, partial lesion rodents and transgenic mice demonstrating progressive nigral degeneration have been and will continue to be assets to the field. Although, rodent models emulating premotor PD are not fully asymptomatic; modest reductions in striatal DA result in cognitive impairments. This mini review article gives a brief summary of SPN dynamics in animal models of PD.

Keywords: Parkinson's disease, striatum, direct pathway, indirect pathway, synaptic plasticity

\section{INTRODUCTION}

Parkinson's disease (PD) is the most common neurodegenerative movement disorder with more than six million patients worldwide (GBD 2016 Parkinson's Disease Collaborators, 2018). PD is largely characterized by the progressive degeneration of substantia nigra pars compacta $(\mathrm{SNc})$ dopamine $(\mathrm{DA})$ neurons that innervate the striatum. The striatum is a critical basal ganglia nucleus involved in initiating and regulating goal-directed movement; in PD, this region undergoes progressive DA depletion leading to the hallmark motor symptoms, i.e., resting tremor, bradykinesia, postural instability, and rigidity. The striatum itself entails a degree of cellular heterogeneity but is primarily dominated by GABAergic spiny projection neurons (SPNs). These SPNs have dendritic arbors heavily populated with spines chiefly consisting of corticostriatal and thalamostriatal circuitry (Bolam et al., 2000; Villalba et al., 2009; Smith et al., 2014). SPNs can 
be subdivided into two distinct populations based on the DA receptor expression profile and projection patterns. SPNs that express D2 DA receptors project to the external segment of the globus pallidus forming the indirect pathway (iSPNs) and SPNs that express D1 DA receptors project to the substantia nigra pars reticulata and internal segment of the globus pallidus forming the direct pathway (dSPNs). DA effects on each SPN population are distinct; in iSPNs D2 receptor activation engages $G_{i / o}$ proteins, decreases intrinsic excitability and promotes long-term depression (LTD); in contrast D1 receptors in dSPNs couple to $G_{\text {olf }}$, increase intrinsic excitability, and promote long-term potentiation (LTP; Surmeier et al., 2007). Animal models of PD have shed light on a number of adaptations that arise in response to DA loss. In this concise review we focus on SPN dynamics in animal models of PD.

\section{SPN DYNAMICS AND TRANSGENIC MODELS}

Multiple genes have been implicated in PD (SNCA, LRRK2, PRKN, PINK1, and PARK7) and incorporated into transgenic rats and/or mice. A consistent finding across transgenic models of PD is impaired synaptic plasticity. Both dSPN and iSPNs are capable of undergoing forms of cellular learning and memory whereby synapses are weakened (LTD) or strengthened (LTP). In the striatum LTD is selective for corticostriatal circuits ( $\mathrm{Wu}$ et al., 2015) and induction is achieved post-synaptically with presynaptic expression that is dependent on endocannabinoid signaling. LTD deficits are consistently reported in multiple transgenic models including PINK1 knockout (Kitada et al., 2007; Madeo et al., 2016), LRRK2 knockout (Chou et al., 2014) and A53T synuclein mouse models (Kurz et al., 2010; Tozzi et al., 2012). It is unclear whether the LTD deficit is specific to iSPNs or dSPNs. Some have argued that only iSPNs are capable of undergoing LTD, and thus the deficit only impacts iSPNs (Kreitzer and Malenka, 2007). However, others report LTD in iSPNs and dSPNs (Wang et al., 2006; Shen et al., 2008; Bagetta et al., 2011; Trusel et al., 2015; Wu et al., 2015). The disparity is likely a consequence of intrastriatal electrical stimulation evoking DA release that stimulates D1 receptors, thereby occluding LTD induction. Consistent with this, DA receptor activation uncovers SPN selectivity for LTD (Wu et al., 2015). The impairment of LTD in parkinsonian transgenic mice is a consequence of diminished D2 receptor stimulation resulting in reduced endocannabinoid production but also decreased CB1 receptor expression (Kurz et al., 2010; Madeo et al., 2016); boosting these impairments through CB1 recepetor agonism, increasing DA, or D2 agonism rescues LTD (Kitada et al., 2007; Chou et al., 2014; Madeo et al., 2016). The ability to rescue with D2 agonism may suggest selectivity for iSPNs; however, D2 receptor activation is also involved in dSPN LTD. D2 stimulation on cholinergic interneurons suppresses M1 receptor signaling to dis-inhibit L-type calcium channels allowing for LTD in dSPNs (Wang et al., 2006; Augustin et al., 2018). Nevertheless, based on the classical basal ganglia model it would hypothesize that this impairment is specific to iSPNs. This model posits that the dSPN circuit is the "go" pathway and the iSPN circuit is the "no-go" pathway;
dSPN activity thus promotes and iSPN activity suppresses motor activity (Surmeier et al., 2007). In this framework it would be expected that the LTD deficit would be selective for iSPNs thereby biasing striatal output in favor of the indirect "no-go" pathway and motor suppression; consistent with this, deletion of D2 receptors, effectively removing DA-mediated inhibition, in iSPNs impairs motor activity (Lemos et al., 2016; Bello et al., 2017) as does optogenetic stimulation of iSPNs (Kravitz et al., 2010). Further supporting indirect pathway dominance in PD, corticostriatal responses in a lesion model are enhanced and diminished in iSPNs and dSPNs, respectively (Flores-Barrera et al., 2010; Escande et al., 2016), and optogenetic stimulation of dSPNs in parkinsonian mice improves motor function (Kravitz et al., 2010). However, more recent studies demonstrate that coordinated motor activity requires concurrent activation of both iSPN and dSPN populations (Cui et al., 2013; Freeze et al., 2013; Tecuapetla et al., 2014) making it less certain whether the LTD deficits would be pathway specific. Generating transgenic lines with SPN cell-type reporters will be necessary to further dissect circuit-specific changes but it is also important to compare with other PD models independent of genetic mutations.

The generation of genetic models has certainly advanced the understanding of PD; however, these models do not fully emulate the disease state and over $90 \%$ of PD cases (late onset) are idiopathic with no known genetic association (Puschmann, 2013). Moreover, transgenic models do not develop neurodegeneration with a few exceptions. In the G2019S LRRK2 model, SNc degeneration $(\sim 40 \%-50 \%)$ is reported at 12-16 months of age (Chen et al., 2012) whereas others report no (Yue et al., 2015; Sloan et al., 2016) or very mild ( 20\%) degeneration (Ramonet et al., 2011) even in 18-21-month-old animals. Reduced tyrosine hydroxylase staining in the $\mathrm{SNc}$ is observed in the $\alpha$-synuclein A53T model at 12 months of age but the magnitude was not quantified (Yamasaki et al., 2016). In contrast, others report no degeneration in A53T mice (Gispert et al., 2003; Kurz et al., 2010). PINK1 animals show no degeneration at 8-9 months (Kitada et al., 2007). Similarly, Parkin deficient animals have no SNc cell loss even at 24 months of age (Goldberg et al., 2003) although animals expressing the Parkin-Q311X mutation show SNc DA neuron loss (40\%) at 16 months of age (Lu et al., 2009). These reports support the notion that genetic perturbations increase the vulnerability of SNc DA neurons but are not sufficient for degeneration. SNc degeneration is also not necessary for parkinsonian symptoms. Neuroleptic treatment results in parkinsonism due to D2 receptor antagonism thereby disinhibiting iSPNs and cholinergic interneurons (Kharkwal et al., 2016); chronic neuroleptic treatment even manifests some of the same functional and anatomical changes (Sebel et al., 2017) observed in PD models (Fieblinger et al., 2014). Transgenic models have also not reproduced the changes in SPN dendritic arborization or spine density.

One of the most consistent and reproducible findings in rodent models, non-human primates, and post mortem tissue from patients is a robust loss of spines on SPNs (McNeill et al., 1988; Ingham et al., 1989; Stephens et al., 2005; Zaja-Milatovic et al., 2005; Day et al., 2006; Villalba et al., 2009; Zhang et al., 2013; 
Anaya-Martínez et al., 2014; Fieblinger et al., 2014; Nishijima et al., 2014; Suárez et al., 2014; Toy et al., 2014; Suarez et al., 2016; Bentea et al., 2017; Gagnon et al., 2017; Gomez et al., 2019); this PD associated anatomical change has not been found (Matikainen-Ankney et al., 2016) or is yet to be reported in transgenic animals with the exception of the Pitx $3^{-/-}$mouse (Suarez et al., 2018). Both dSPNs and iSPNs of Pitx $3^{-/-}$mice have decreased spine density. However, Pitx3 is not a gene associated with PD; it is involved in the development of DA neurons. In Pitx $3^{-/-}$animals, most SNc DA neurons fail to fully differentiate and do not innervate the striatum (Hwang et al., 2003). Thus it is rather a model with neurodevelopmental deficits as opposed to a parkinsonian model of degeneration. The absence of spine pruning in transgenic models of PD may relate to the fact that spine pruning seems to be associated with the severity of DA loss (Villalba et al., 2009; Suárez et al., 2014) and sufficient depletion may not be reached in transgenic mice. The models that reproducibly induce robust degeneration of SNc DA neurons are the toxin models. Toxin models such as the 6-OHDA and MPTP models of PD are far from perfect but comparison with evidence from transgenic models provides a means to determine what is consistent across PD models and thus most likely to be translatable to the human condition.

\section{SPN DYNAMICS AND TOXIN MODELS}

6-OHDA produces a near complete degeneration of DA neurons when injected into the medial forebrain bundle or SNc whereas the same result can be achieved with systemic injection of MPTP. Both toxins produce a rapid and robust loss of DA neurons with overt motor impairment that can be ameliorated by levodopa administration. Consistent with evidence from human post-mortem tissue (McNeill et al., 1988; Stephens et al., 2005; Zaja-Milatovic et al., 2005), both MPTP and 6-OHDA lesioned non-human primates and rodents (Ingham et al., 1989; Day et al., 2006; Villalba et al., 2009; Zhang et al., 2013; Anaya-Martínez et al., 2014; Fieblinger et al., 2014; Nishijima et al., 2014; Suárez et al., 2014; Toy et al., 2014; Ueno et al., 2014; Suarez et al., 2016; Gagnon et al., 2017; Graves and Surmeier, 2019) show reduced SPN dendritic arborization and robust spine pruning in the striatum. However, whether spine pruning is selective for iSPN or dSPNs is debatable. Some report selective spine pruning in iSPNs (Day et al., 2006; Fieblinger et al., 2014; Nishijima et al., 2014); others report decreased spine density in both iSPNs and dSPNs (Villalba et al., 2009; Suárez et al., 2014; Toy et al., 2014; Suarez et al., 2016; Gagnon et al., 2017; Gomez et al., 2019) and it remains to be seen what might account for the disparate findings. One potential variable is the time post-lesion at which investigations are conducted; dSPN spine density is unchanged 30 days post-6-OHDA lesion but decreased 60 days post-lesion (Graves and Surmeier, 2019). The spines of both iSPNs and dSPNs are the sites of glutamatergic axospinous circuitry; this is predominantly corticostriatal circuitry with the remaining subset of axospinous synapses belonging to thalamostriatal circuits. Spine pruning primarily reflects a loss of corticostriatal axospinous synapses (Zhang et al., 2013; Fieblinger et al., 2014; Bentea et al., 2017). Paradoxically, lesioning the motor cortex prevents and even reverses spine loss in 6-OHDA lesioned rats, an effect also achieved by mGluR2/3 antagonism (Garcia et al., 2010). In iSPNs M1 receptors increase dendritic excitability and contribute to spine pruning (Shen et al., 2007). In contrast, M4 signaling in dSPNs promotes LTD at axospinous synapses (Shen et al., 2015) but it is unclear whether this modulates dSPN spine dynamics in PD models. While the transgenic and toxin models disagree when it comes to SPN anatomical changes, consensus is found with synaptic plasticity. Similar to findings in transgenic models, LTD is impaired in both MPTP treated mice (Chen et al., 2008) and monkeys (Quik et al., 2006). LTD is also impaired in iSPNs from reserpinized and 6-OHDA lesioned mice (Kreitzer and Malenka, 2007; Shen et al., 2008; Thiele et al., 2014); rescuing iSPN LTD improves motor impairments (Kreitzer and Malenka, 2007). Based on the above data the LTD impairments in transgenic models are also likely specific to iSPNs. In contrast, LTP is impaired in dSPNs, consistent with a dis-inhibition of cholinergic interneurons and increased M4 stimulation; inhibition of cholinergic interneurons, M4 antagonism, or D1 agonism improves motor performance (Shen et al., 2008; Paille et al., 2010; Thiele et al., 2014; Ztaou et al., 2016). Agonism at D2 receptors also restores impaired LTD in iSPNs similar to the restoration in transgenic mice, whereas D1 receptor agonism restores LTP in dSPNs (Shen et al., 2008); restoring plasticity impairments in both SPN populations also improves motor function (Picconi et al., 2003; Thiele et al., 2014; Trusel et al., 2015).

In addition to spine pruning and impairments of synaptic plasticity, there is a rearrangement of excitatory synapse machinery. MPTP lesioning increases calcium permeable AMPA receptors in iSPNs (VanLeeuwen et al., 2010; Kintz et al., 2013). This is particularly intriguing given the problems with synaptic plasticity in parkinsonian animals; incorporation of calcium permeable AMPA receptors allows for an NMDA-independent form of LTP (Mameli et al., 2011). In this framework iSPNs would have a lower threshold to induce LTP and without sufficient DA to induce LTD this would contribute to indirect pathway dominance.

\section{SPN DYNAMICS PRECEDING MOTOR DYSFUNCTION}

By the time patients with PD are diagnosed and experiencing motor symptoms, $>50 \%$ of SNc DA neurons have been lost and striatal levels of DA reduced by $>60 \%$ (Tissingh et al., 1998; Hilker et al., 2005; Cheng et al., 2010). The premotor/prodromal phase is estimated to consist of a 4-6 year period prior to the emergence of motor symptoms and diagnosis (Fearnley and Lees, 1991; Morrish et al., 1998; Marek et al., 2001). During this premotor phase there are likely a number of adaptations occurring within the striatum to compensate for the loss of DA neurons. Human data demonstrate decreased DA reuptake and enhanced DA synthesis in early PD (Nandhagopal et al., 2011); how circuit function is altered and whether alterations are compensatory or pathological is much less clear and an important gap that needs to be addressed. Several groups have made efforts to investigate the premotor phase of PD 
either through transgenic strategies or partial lesioning of the nigrostriatal system.

PINK1 knockout heterozygous mice are motorically asymptomatic despite having $\sim 40 \%$ reduction in extracellular DA (Madeo et al., 2014). These animals exhibit a selective deficit in LTP that is restored by drugs that increase striatal DA including amphetamine, tyramine and selegiline and partially restored by levodopa but not a D1 agonist (Madeo et al., 2014); however, D1 agonsim does restore LTP in homozygous knockout mice (Kitada et al., 2007). In partial 6-OHDA lesioned rats with modest motor impairments, LTP deficits and motor symptoms are also rescued by D1 agonism (Paille et al., 2010). It is unclear why in heterozygous PINK1 knockout LTP is not rescued by $\mathrm{D} 1$ stimulation but data support a DA-dependent deficit. Given that LTP in iSPNs utilizes $A_{2 A}$ receptors for LTP and D2 receptors promote LTD, it is likely that the LTP deficit is dSPN specific. If this is the case it indicates that the balance between iSPN and dSPN circuit function manifests well before motor symptoms. Consistent with this, in animals that receive a partial 6-OHDA lesion and do not present with motor dysfunction, there is a reduced response in dSPNs to cortical stimulation (Escande et al., 2016). In mice with motor impairment, iSPN corticostriatal responses are increased and dSPN corticostriatal responses further reduced (Escande et al., 2016). Taken together this suggests that dSPN circuits are the first to be impacted by moderate DA depletion and although there are not motor symptoms, animals with partial DA depletion show anxiety- and depression-like behaviors, as well as cognitive impairments (Tadaiesky et al., 2008, 2010; Bonito-Oliva et al., 2014a,b; Tozzi et al., 2016; Loiodice et al., 2018; Ztaou et al., 2018).

The 6-OHDA partial lesion model with 40\% DA depletion exhibits impaired spatial object recognition (Ztaou et al., 2018) but with $75 \%$ DA depletion, novel object recognition is also impaired (Bonito-Oliva et al., 2014b; Loiodice et al., 2018). Hippocampal circuitry contributes to spatial and recognition memory and with partial lesions, hippocampal DA is reduced and hippocampal LTP impaired (Bonito-Oliva et al., 2014b). Levodopa or D1 agonism, but not D2 agonism rescues the deficits in novel object recognition and hippocampal LTP (Bonito-Oliva et al., 2014b; Loiodice et al., 2018) suggesting that early dysfunction not only occurs in dSPN circuits but are manifesting in D1-dependent systems that extend beyond the striatum. Similar cognitive deficits have also been observed in alpha-synuclein overexpression (Subramaniam et al., 2018). MitoPark mice have progressive degeneration of DA neurons (Ekstrand et al., 2007) with motor impairment beginning at 12 weeks of age (Galter et al., 2010). Preceding the onset of motor symptoms, 8-week-old MitoPark mice

\section{REFERENCES}

Anaya-Martínez, V., Gutierrez-Valdez, A. L., Ordoñez-Librado, J. L., MontielFlores, E., Sánchez-Betancourt, J., Sánchez Vázquez del Mercado, C., et al. (2014). The presence of perforated synapses in the striatum after dopamine depletion, is this a sign of maladaptive brain plasticity? Microscopy 63, 427-435. doi: $10.1093 /$ jmicro/dfu032 also perform poorly in the novel object recognition test and have impaired learning in a spatial task ( $\mathrm{Li}$ et al., 2013). These cognitive impairments arise at a time when there is a DA deficit but well before the emergence of motor dysfunction.

Impaired striatal function may contribute to impaired spatial learning. MPTP partial lesioned animals are impaired in learning the constant-start version of the water maze task, which involves a cue-dependent navigation strategy within the striatum but performance is unaltered using a variable-start version of the task, which is more dependent on a hippocampal spatial navigation strategy (Da Cunha et al., 2006). The spatial task employed by Li et al. (2013) also used a constant start location paradigm. Therefore, the learning deficit could reflect a striatal dysfunction due to reduced D1 signaling in dSPNs.

\section{CONCLUSION AND FUTURE DIRECTIONS}

There is no perfect model system to study PD; by examining key findings from the diverse set of preclinical models two themes emerge. First, motorically impaired PD models have impaired synaptic function, particularly in the form of LTP and LTD and these impairments are cell-type specific with LTP being impaired in dSPNs and LTD impaired in iSPNs. Second, dSPNs appear to be the first SPN population to undergo functional alterations in response to moderate DA depletion. At this disease stage subjects are not motorically impaired but are cognitively impaired which may be a combination of dysfunction in dSPN circuits as well as extrastriatal D1 systems. Moving forward it will be important to better investigate changes during premotor phases to understand how the striatum and other DA reliant circuits adapt and deteriorate during disease progression. Moreover, it will be critical to determine what adaptations contribute to motor and non-motor symptoms and which, if any, are fighting to maintain normative function so that treatment strategies can be designed to directly target maladaptive plasticity and support or strengthen the compensatory changes.

\section{AUTHOR CONTRIBUTIONS}

SG and YD drafted and edited the manuscript. Both authors approved the final manuscript.

\section{ACKNOWLEDGMENTS}

We wish to thank the University of Minnesota Department of Pharmacology and Institute for Translational Neuroscience for financial support.

Augustin, S. M., Chancey, J. H., and Lovinger, D. M. (2018). Dual dopaminergic regulation of corticostriatal plasticity by cholinergic interneurons and indirect pathway medium spiny neurons. Cell Rep. 24, 2883-2893. doi: 10.1016/j.celrep. 2018.08.042

Bagetta, V., Picconi, B., Marinucci, S., Sgobio, C., Pendolino, V., Ghiglieri, V., et al. (2011). Dopamine-dependent long-term depression is expressed in striatal spiny neurons of both direct and indirect pathways: implications for 
Parkinson's disease. J. Neurosci. 31, 12513-12522. doi: 10.1523/JNEUROSCI. 2236-11.2011

Bello, E. P., Casas-Cordero, R., Galiñanes, G. L., Casey, E., Belluscio, M. A., Rodriguez, V., et al. (2017). Inducible ablation of dopamine D2 receptors in adult mice impairs locomotion, motor skill learning and leads to severe parkinsonism. Mol. Psychiatry 22, 595-604. doi: 10.1038/mp.20 16.105

Bentea, E., Moore, C., Deneyer, L., Verbruggen, L., Churchill, M. J., Hood, R. L., et al. (2017). Plastic changes at corticostriatal synapses predict improved motor function in a partial lesion model of Parkinson's disease. Brain Res. Bull. 130, 257-267. doi: 10.1016/j.brainresbull.2017.02.005

Bolam, J. P., Hanley, J. J., Booth, P. A., and Bevan, M. D. (2000). Synaptic organisation of the basal ganglia. J. Anat. 196, 527-542. doi: 10.1046/j.14697580.2000.19640527.x

Bonito-Oliva, A., Masini, D., and Fisone, G. (2014a). A mouse model of non-motor symptoms in Parkinson's disease: focus on pharmacological interventions targeting affective dysfunctions. Front. Behav. Neurosci. 8:290. doi: 10.3389/fnbeh.2014.00290

Bonito-Oliva, A., Pignatelli, M., Spigolon, G., Yoshitake, T., Seiler, S., Longo, F., et al. (2014b). Cognitive impairment and dentate gyrus synaptic dysfunction in experimental parkinsonism. Biol. Psychiatry 75, 701-710. doi: 10.1016/j. biopsych.2013.02.015

Chen, Y. H., Harvey, B. K., Hoffman, A. F., Wang, Y., Chiang, Y. H., and Lupica, C. R. (2008). MPTP-induced deficits in striatal synaptic plasticity are prevented by glial cell line-derived neurotrophic factor expressed via an adenoassociated viral vector. FASEB J. 22, 261-275. doi: 10.1096/fj.07-8797com

Chen, C. Y., Weng, Y. H., Chien, K. Y., Lin, K. J., Yeh, T. H., Cheng, Y. P., et al. (2012). (G2019S) LRRK2 activates MKK4-JNK pathway and causes degeneration of $\mathrm{SN}$ dopaminergic neurons in a transgenic mouse model of PD. Cell Death Differ. 19, 1623-1633. doi: 10.1038/cdd.2012.42

Cheng, H. C., Ulane, C. M., and Burke, R. E. (2010). Clinical progression in Parkinson disease and the neurobiology of axons. Ann. Neurol. 67, 715-725. doi: 10.1002/ana.21995

Chou, J. S., Chen, C. Y., Chen, Y. L., Weng, Y. H., Yeh, T. H., Lu, C. S., et al. (2014). (G2019S) LRRK2 causes early-phase dysfunction of SNpc dopaminergic neurons and impairment of corticostriatal long-term depression in the PD transgenic mouse. Neurobiol. Dis. 68, 190-199. doi: 10.1016/j.nbd.2014.04.021

Cui, G., Jun, S. B., Jin, X., Pham, M. D., Vogel, S. S., Lovinger, D. M., et al. (2013). Concurrent activation of striatal direct and indirect pathways during action initiation. Nature 494, 238-242. doi: 10.1038/nature11846

Da Cunha, C., Silva, M. H., Wietzikoski, S., Wietzikoski, E. C., Ferro, M. M., Kouzmine, I., et al. (2006). Place learning strategy of substantia nigra pars compacta-lesioned rats. Behav. Neurosci. 120, 1279-1284. doi: 10.1037/07357044.120.6.1279

Day, M., Wang, Z., Ding, J., An, X., Ingham, C. A., Shering, A. F., et al. (2006). Selective elimination of glutamatergic synapses on striatopallidal neurons in Parkinson disease models. Nat. Neurosci. 9, 251-259. doi: 10.1038/nn1632

Ekstrand, M. I., Terzioglu, M., Galter, D., Zhu, S., Hofstetter, C., Lindqvist, E., et al. (2007). Progressive parkinsonism in mice with respiratory-chaindeficient dopamine neurons. Proc. Natl. Acad. Sci. U S A 104, 1325-1330. doi: 10.1073/pnas.0605208103

Escande, M. V., Taravini, I. R., Zold, C. L., Belforte, J. E., and Murer, M. G. (2016). Loss of homeostasis in the direct pathway in a mouse model of asymptomatic Parkinson's disease. J. Neurosci. 36, 5686-5698. doi: 10.1523/JNEUROSCI. 0492-15.2016

Fearnley, J. M., and Lees, A. J. (1991). Ageing and Parkinson's disease: substantia nigra regional selectivity. Brain 114, 2283-2301. doi: 10.1093/brain/114.5.2283

Fieblinger, T., Graves, S. M., Sebel, L. E., Alcacer, C., Plotkin, J. L., Gertler, T. S., et al. (2014). Cell type-specific plasticity of striatal projection neurons in parkinsonism and L-DOPA-induced dyskinesia. Nat. Commun. 5:5316. doi: $10.1038 /$ ncomms6316

Flores-Barrera, E., Vizcarra-Chacón, B. J., Tapia, D., Bargas, J., and Galarraga, E. (2010). Different corticostriatal integration in spiny projection neurons from direct and indirect pathways. Front. Syst. Neurosci. 4:15. doi: 10.3389/fnsys. 2010.00015

Freeze, B. S., Kravitz, A. V., Hammack, N., Berke, J. D., and Kreitzer, A. C. (2013). Control of basal ganglia output by direct and indirect pathway projection neurons. J. Neurosci. 33, 18531-18539. doi: 10.1523/JNEUROSCI.1278-13.2013
Gagnon, D., Petryszyn, S., Sanchez, M. G., Bories, C., Beaulieu, J. M., De Koninck, Y., et al. (2017). Striatal neurons expressing $D_{1}$ and $D_{2}$ receptors are morphologically distinct and differently affected by dopamine denervation in mice. Sci. Rep. 7:41432. doi: 10.1038/srep41432

Galter, D., Pernold, K., Yoshitake, T., Lindqvist, E., Hoffer, B., Kehr, J., et al. (2010). MitoPark mice mirror the slow progression of key symptoms and L-DOPA response in Parkinson's disease. Genes Brain Behav. 9, 173-181. doi: 10.1111/j. 1601-183x.2009.00542.x

Garcia, B. G., Neely, M. D., and Deutch, A. Y. (2010). Cortical regulation of striatal medium spiny neuron dendritic remodeling in parkinsonism: modulation of glutamate release reverses dopamine depletion-induced dendritic spine loss. Cereb. Cortex 20, 2423-2432. doi: 10.1093/cercor/bhp317

GBD 2016 Parkinson's Disease Collaborators. (2018). Global, regional, and national burden of Parkinson's disease, 1990-2016: a systematic analysis for the Global Burden of Disease Study 2016. Lancet Neurol. 17, 939-953. doi: 10.1016/S1474-4422(18)30295-3

Gispert, S., Del Turco, D., Garrett, L., Chen, A., Bernard, D. J., Hamm-Clement, J., et al. (2003). Transgenic mice expressing mutant A53T human $\alpha$-synuclein show neuronal dysfunction in the absence of aggregate formation. Mol. Cell. Neurosci. 24, 419-429. doi: 10.1016/s1044-7431(03)00198-2

Goldberg, M. S., Fleming, S. M., Palacino, J. J., Cepeda, C., Lam, H. A., Bhatnagar, A., et al. (2003). Parkin-deficient mice exhibit nigrostriatal deficits but not loss of dopaminergic neurons. J. Biol. Chem. 278, 43628-43635. doi: $10.1074 /$ jbc.M308947200

Gomez, G., Escande, M. V., Suarez, L. M., Rela, L., Belforte, J. E., Moratalla, R., et al. (2019). Changes in dendritic spine density and inhibitory perisomatic connectivity onto medium spiny neurons in L-Dopa-induced dyskinesia. Mol. Neurobiol. doi: 10.1007/s12035-019-1515-4 [Epub ahead of print].

Graves, S. M., and Surmeier, D. J. (2019). Delayed spine pruning of direct pathway spiny projection neurons in a mouse model of Parkinson's disease. Front. Cell. Neurosci. 13:32. doi: 10.3389/fncel.2019.00032

Hilker, R., Schweitzer, K., Coburger, S., Ghaemi, M., Weisenbach, S., Jacobs, A. H., et al. (2005). Nonlinear progression of Parkinson disease as determined by serial positron emission tomographic imaging of striatal fluorodopa $\mathrm{F}$ 18 activity. Arch. Neurol. 62, 378-382. doi: 10.1001/archneur.62.3.378

Hwang, D. Y., Ardayfio, P., Kang, U. J., Semina, E. V., and Kim, K. S. (2003). Selective loss of dopaminergic neurons in the substantia nigra of Pitx3deficient aphakia mice. Mol. Brain Res. 114, 123-131. doi: 10.1016/s0169$328 \mathrm{x}(03) 00162-1$

Ingham, C. A., Hood, S. H., and Arbuthnott, G. W. (1989). Spine density on neostriatal neurones changes with 6-hydroxydopamine lesions and with age. Brain Res. 503, 334-338. doi: 10.1016/0006-8993(89)91686-7

Kharkwal, G., Brami-Cherrier, K., Lizardi-Ortiz, J. E., Nelson, A. B., Ramos, M., Del Barrio, D., et al. (2016). Parkinsonism driven by antipsychotics originates from dopaminergic control of striatal cholinergic interneurons. Neuron 91, 67-78. doi: 10.1016/j.neuron.2016.06.014

Kintz, N., Petzinger, G. M., Akopian, G., Ptasnik, S., Williams, C., Jakowec, M. W., et al. (2013). Exercise modifies $\alpha$-amino-3-hydroxy-5methyl-4-isoxazolepropionic acid receptor expression in striatopallidal neurons in the 1-methyl-4-phenyl-1,2,3,6-tetrahydropyridine-lesioned mouse. J. Neurosci. Res. 91, 1492-1507. doi: 10.1002/jnr.23260

Kitada, T., Pisani, A., Porter, D. R., Yamaguchi, H., Tscherter, A., Martella, G., et al. (2007). Impaired dopamine release and synaptic plasticity in the striatum of PINK1-deficient mice. Proc. Natl. Acad. Sci. U S A 104, 11441-11446. doi: 10.1073/pnas.0702717104

Kravitz, A. V., Freeze, B. S., Parker, P. R., Kay, K., Thwin, M. T., Deisseroth, K., et al. (2010). Regulation of parkinsonian motor behaviours by optogenetic control of basal ganglia circuitry. Nature 466, 622-626. doi: 10.1038/nature09159

Kreitzer, A. C., and Malenka, R. C. (2007). Endocannabinoid-mediated rescue of striatal LTD and motor deficits in Parkinson's disease models. Nature 445, 643-647. doi: 10.1038/nature05506

Kurz, A., Double, K. L., Lastres-Becker, I., Tozzi, A., Tantucci, M., Bockhart, V., et al. (2010). A53T- $\alpha$-synuclein overexpression impairs dopamine signaling and striatal synaptic plasticity in old mice. PLoS One 5:e11464. doi: 10.1371/journal.pone.0011464

Lemos, J. C., Friend, D. M., Kaplan, A. R., Shin, J. H., Rubinstein, M., Kravitz, A. V., et al. (2016). Enhanced GABA transmission drives bradykinesia following loss 
of dopamine D2 receptor signaling. Neuron 90, 824-838. doi: 10.1016/j.neuron. 2016.04.040

Li, X., Redus, L., Chen, C., Martinez, P. A., Strong, R., Li, S., et al. (2013). Cognitive dysfunction precedes the onset of motor symptoms in the MitoPark mouse model of Parkinson's disease. PLoS One 8:e71341. doi: 10.1371/journal.pone. 0071341

Loiodice, S., Wing Young, H., Rion, B., Méot, B., Montagne, P., Denibaud, A. S., et al. (2018). Implication of nigral dopaminergic lesion and repeated L-dopa exposure in neuropsychiatric symptoms of Parkinson's disease. Behav. Brain Res. 360, 120-127. doi: 10.1016/j.bbr.2018.12.007

Lu, X. H., Fleming, S. M., Meurers, B., Ackerson, L. C., Mortazavi, F., Lo, V., et al. (2009). Bacterial artificial chromosome transgenic mice expressing a truncated mutant parkin exhibit age-dependent hypokinetic motor deficits, dopaminergic neuron degeneration, and accumulation of proteinase K-resistant $\alpha$-synuclein. J. Neurosci. 29, 1962-1976. doi: 10.1523/JNEUROSCI. 5351-08.2009

Madeo, G., Schirinzi, T., Maltese, M., Martella, G., Rapino, C., Fezza, F., et al. (2016). Dopamine-dependent CB1 receptor dysfunction at corticostriatal synapses in homozygous PINK1 knockout mice. Neuropharmacology 101, 460-470. doi: 10.1016/j.neuropharm.2015.10.021

Madeo, G., Schirinzi, T., Martella, G., Latagliata, E. C., Puglisi, F., Shen, J., et al. (2014). PINK1 heterozygous mutations induce subtle alterations in dopaminedependent synaptic plasticity. Mov. Disord. 29, 41-53. doi: 10.1002/mds.25724

Mameli, M., Bellone, C., Brown, M. T., and Lüscher, C. (2011). Cocaine inverts rules for synaptic plasticity of glutamate transmission in the ventral tegmental area. Nat. Neurosci. 14, 414-416. doi: 10.1038/nn.2763

Marek, K., Innis, R., van Dyck, C., Fussell, B., Early, M., Eberly, S., et al. (2001). [123I] $\beta$-CIT SPECT imaging assessment of the rate of Parkinson's disease progression. Neurology 57, 2089-2094. doi: 10.1212/wnl.57.11.2089

Matikainen-Ankney, B. A., Kezunovic, N., Mesias, R. E., Tian, Y., Williams, F. M., Huntley, G. W., et al. (2016). Altered development of synapse structure and function in striatum caused by Parkinson's disease-Linked LRRK2-G2019S mutation. J. Neurosci. 36, 7128-7141. doi: 10.1523/JNEUROSCI.3314-15.2016

McNeill, T. H., Brown, S. A., Rafols, J. A., and Shoulson, I. (1988). Atrophy of medium spiny I striatal dendrites in advanced Parkinson's disease. Brain Res. 455, 148-152. doi: 10.1016/0006-8993(88)90124-2

Morrish, P. K., Rakshi, J. S., Bailey, D. L., Sawle, G. V., and Brooks, D. J. (1998). Measuring the rate of progression and estimating the preclinical period of Parkinson's disease with [18F]dopa PET. J. Neurol. Neurosurg. Psychiatry 64, 314-319. doi: 10.1136/jnnp.64.3.314

Nandhagopal, R., Kuramoto, L., Schulzer, M., Mak, E., Cragg, J., McKenzie, J., et al. (2011). Longitudinal evolution of compensatory changes in striatal dopamine processing in Parkinson's disease. Brain 134, 3290-3298. doi: 10.1093/brain/awr233

Nishijima, H., Suzuki, S., Kon, T., Funamizu, Y., Ueno, T., Haga, R., et al. (2014). Morphologic changes of dendritic spines of striatal neurons in the levodopainduced dyskinesia model. Mov. Disord. 29, 336-343. doi: 10.1002/mds.25826

Paille, V., Picconi, B., Bagetta, V., Ghiglieri, V., Sgobio, C., Di Filippo, M., et al. (2010). Distinct levels of dopamine denervation differentially alter striatal synaptic plasticity and NMDA receptor subunit composition. J. Neurosci. 30, 14182-14193. doi: 10.1523/JNEUROSCI.2149-10.2010

Picconi, B., Centonze, D., Hakansson, K., Bernardi, G., Greengard, P., Fisone, G., et al. (2003). Loss of bidirectional striatal synaptic plasticity in L-DOPAinduced dyskinesia. Nat. Neurosci. 6, 501-506. doi: 10.1038/nn1040

Puschmann, A. (2013). Monogenic Parkinson's disease and parkinsonism: clinical phenotypes and frequencies of known mutations. Parkinsonism. Relat. Disord. 19, 407-415. doi: 10.1016/j.parkreldis.2013.01.020

Quik, M., Parameswaran, N., McCallum, S. E., Bordia, T., Bao, S., McCormack, A., et al. (2006). Chronic oral nicotine treatment protects against striatal degeneration in MPTP-treated primates. J. Neurochem. 98, 1866-1875. doi: 10.1111/j.1471-4159.2006.04078.x

Ramonet, D., Daher, J. P., Lin, B. M., Stafa, K., Kim, J., Banerjee, R., et al. (2011). Dopaminergic neuronal loss, reduced neurite complexity and autophagic abnormalities in transgenic mice expressing G2019S mutant LRRK2. PLoS One 6:e18568. doi: 10.1371/journal.pone.0018568

Sebel, L. E., Graves, S. M., Chan, C. S., and Surmeier, D. J. (2017). Haloperidol selectively remodels striatal indirect pathway circuits. Neuropsychopharmacology 42, 963-973. doi: 10.1038/npp.2016.173
Shen, W., Flajolet, M., Greengard, P., and Surmeier, D. J. (2008). Dichotomous dopaminergic control of striatal synaptic plasticity. Science 321, 848-851. doi: 10.1126/science.1160575

Shen, W., Plotkin, J. L., Francardo, V., Ko, W. K., Xie, Z., Li, Q., et al. (2015). M4 muscarinic receptor signaling ameliorates striatal plasticity deficits in models of L-DOPA-induced dyskinesia. Neuron 88, 762-773. doi: 10.1016/j. neuron.2015.10.039

Shen, W., Tian, X., Day, M., Ulrich, S., Tkatch, T., Nathanson, N. M., et al. (2007). Cholinergic modulation of Kir2 channels selectively elevates dendritic excitability in striatopallidal neurons. Nat. Neurosci. 10, 1458-1466. doi: $10.1038 / \mathrm{nn} 1972$

Sloan, M., Alegre-Abarrategui, J., Potgieter, D., Kaufmann, A. K., Exley, R., Deltheil, T., et al. (2016). LRRK2 BAC transgenic rats develop progressive, L-DOPA-responsive motor impairment, and deficits in dopamine circuit function. Hum. Mol. Genet. 25, 951-963. doi: 10.1093/hmg/ddv628

Smith, Y., Galvan, A., Ellender, T. J., Doig, N., Villalba, R. M., Huerta-Ocampo, I., et al. (2014). The thalamostriatal system in normal and diseased states. Front. Syst. Neurosci. 8:5. doi: 10.3389/fnsys.2014.00005

Stephens, B., Mueller, A. J., Shering, A. F., Hood, S. H., Taggart, P., Arbuthnott, G. W., et al. (2005). Evidence of a breakdown of corticostriatal connections in Parkinson's disease. Neuroscience 132, 741-754. doi: 10.1016/j. neuroscience.2005.01.007

Suarez, L. M., Alberquilla, S., García-Montes, J. R., and Moratalla, R. (2018). Differential synaptic remodeling by dopamine in direct and indirect striatal projection neurons in Pitx $3^{-/-}$Mice, a genetic model of Parkinson's disease. J. Neurosci. 38, 3619-3630. doi: 10.1523/JNEUROSCI.3184-17.2018

Suarez, L. M., Solis, O., Aguado, C., Lujan, R., and Moratalla, R. (2016). LDOPA oppositely regulates synaptic strength and spine morphology in D1 and D2 striatal projection neurons in dyskinesia. Cereb. Cortex 26, 4253-4264. doi: 10.1093/cercor/bhw263

Suárez, L. M., Solís, O., Caramés, J. M., Taravini, I. R., Solís, J. M., Murer, M. G., et al. (2014). L-DOPA treatment selectively restores spine density in dopamine receptor D2-expressing projection neurons in dyskinetic mice. Biol. Psychiatry 75, 711-722. doi: 10.1016/j.biopsych.2013.05.006

Subramaniam, S. R., Magen, I., Bove, N., Zhu, C., Lemesre, V., Dutta, G., et al. (2018). Chronic nicotine improves cognitive and social impairment in mice overexpressing wild type $\alpha$-synuclein. Neurobiol. Dis. 117, 170-180. doi: 10.1016/j.nbd.2018.05.018

Surmeier, D. J., Ding, J., Day, M., Wang, Z., and Shen, W. (2007). D1 and D2 dopamine-receptor modulation of striatal glutamatergic signaling in striatal medium spiny neurons. Trends Neurosci. 30, 228-235. doi: 10.1016/j.tins.2007. 03.008

Tadaiesky, M. T., Dombrowski, P. A., Da Cunha, C., and Takahashi, R. N. (2010). Effects of SR141716A on cognitive and depression-related behavior in an animal model of premotor Parkinson's disease. Parkinsons Dis. 2010:238491. doi: 10.4061/2010/238491

Tadaiesky, M. T., Dombrowski, P. A., Figueiredo, C. P., Cargnin-Ferreira, E., Da Cunha, C., and Takahashi, R. N. (2008). Emotional, cognitive and neurochemical alterations in a premotor stage model of Parkinson's disease. Neuroscience 156, 830-840. doi: 10.1016/j.neuroscience.2008.08.035

Tecuapetla, F., Matias, S., Dugue, G. P., Mainen, Z. F., and Costa, R. M. (2014). Balanced activity in basal ganglia projection pathways is critical for contraversive movements. Nat. Commun. 5:4315. doi: 10.1038/ncomms5315

Thiele, S. L., Chen, B., Lo, C., Gertler, T. S., Warre, R., Surmeier, J. D., et al. (2014). Selective loss of bi-directional synaptic plasticity in the direct and indirect striatal output pathways accompanies generation of parkinsonism and L-DOPA induced dyskinesia in mouse models. Neurobiol. Dis. 71, 334-344. doi: 10.1016/j.nbd.2014.08.006

Tissingh, G., Bergmans, P., Booij, J., Winogrodzka, A., van Royen, E. A., Stoof, J. C., et al. (1998). Drug-naive patients with Parkinson's disease in Hoehn and Yahr stages I and II show a bilateral decrease in striatal dopamine transporters as revealed by $\left[{ }^{123 I}\right] \beta$-CIT SPECT. J. Neurol. 245, 14-20. doi: 10.1007/s004150050168

Toy, W. A., Petzinger, G. M., Leyshon, B. J., Akopian, G. K., Walsh, J. P., Hoffman, M. V., et al. (2014). Treadmill exercise reverses dendritic spine loss in direct and indirect striatal medium spiny neurons in the 1-methyl4-phenyl-1,2,3,6-tetrahydropyridine (MPTP) mouse model of Parkinson's disease. Neurobiol. Dis. 63, 201-209. doi: 10.1016/j.nbd.2013.11.017 
Tozzi, A., Costa, C., Siliquini, S., Tantucci, M., Picconi, B., Kurz, A., et al. (2012). Mechanisms underlying altered striatal synaptic plasticity in old A53T- $\alpha$ synuclein overexpressing mice. Neurobiol. Aging 33, 1792-1799. doi: 10.1016/j. neurobiolaging.2011.05.002

Tozzi, A., de Iure, A., Bagetta, V., Tantucci, M., Durante, V., Quiroga-Varela, A., et al. (2016). $\alpha$-synuclein produces early behavioral alterations via striatal cholinergic synaptic dysfunction by interacting With GluN2D N-methylD-aspartate receptor subunit. Biol. Psychiatry 79, 402-414. doi: 10.1016/j. biopsych.2015.08.013

Trusel, M., Cavaccini, A., Gritti, M., Greco, B., Saintot, P. P., Nazzaro, C., et al. (2015). Coordinated regulation of synaptic plasticity at striatopallidal and striatonigral neurons orchestrates motor control. Cell Rep. 13, 1353-1365. doi: 10.1016/j.celrep.2015.10.009

Ueno, T., Yamada, J., Nishijima, H., Arai, A., Migita, K., Baba, M., et al. (2014). Morphological and electrophysiological changes in intratelencephalic-type pyramidal neurons in the motor cortex of a rat model of levodopainduced dyskinesia. Neurobiol. Dis. 64, 142-149. doi: 10.1016/j.nbd.2013. 12.014

VanLeeuwen, J. E., Petzinger, G. M., Walsh, J. P., Akopian, G. K., Vuckovic, M., and Jakowec, M. W. (2010). Altered AMPA receptor expression with treadmill exercise in the 1-methyl-4-phenyl-1,2,3,6-tetrahydropyridine-lesioned mouse model of basal ganglia injury. J. Neurosci. Res. 88, 650-668. doi: 10.1002/jnr. 22216

Villalba, R. M., Lee, H., and Smith, Y. (2009). Dopaminergic denervation and spine loss in the striatum of MPTP-treated monkeys. Exp. Neurol. 215, 220-227. doi: 10.1016/j.expneurol.2008.09.025

Wang, Z., Kai, L., Day, M., Ronesi, J., Yin, H. H., Ding, J., et al. (2006). Dopaminergic control of corticostriatal long-term synaptic depression in medium spiny neurons is mediated by cholinergic interneurons. Neuron 50, 443-452. doi: 10.1016/j.neuron.2006.04.010

Wu, Y. W., Kim, J. I., Tawfik, V. L., Lalchandani, R. R., Scherrer, G., and Ding, J. B. (2015). Input- and cell-type-specific endocannabinoid-dependent LTD in the striatum. Cell Rep. 10, 75-87. doi: 10.1016/j.celrep.2014.12.005

Yamasaki, T., Fujinaga, M., Kawamura, K., Furutsuka, K., Nengaki, N., Shimoda, Y., et al. (2016). Dynamic changes in striatal mGluR1 but not
mGluR5 during pathological progression of Parkinson's disease in human $\alpha$ synuclein A53T transgenic rats: a multi-PET imaging study. J. Neurosci. 36, 375-384. doi: 10.1523/JNEUROSCI.2289-15.2016

Yue, M., Hinkle, K. M., Davies, P., Trushina, E., Fiesel, F. C., Christenson, T. A., et al. (2015). Progressive dopaminergic alterations and mitochondrial abnormalities in LRRK2 G2019S knock-in mice. Neurobiol. Dis. 78, 172-195. doi: 10.1016/j.nbd.2015.02.031

Zaja-Milatovic, S., Milatovic, D., Schantz, A. M., Zhang, J., Montine, K. S., Samii, A., et al. (2005). Dendritic degeneration in neostriatal medium spiny neurons in Parkinson disease. Neurology 64, 545-547. doi: 10.1212/01.wnl. 0000150591.33787.a4

Zhang, Y., Meredith, G. E., Mendoza-Elias, N., Rademacher, D. J., Tseng, K. Y., and Steece-Collier, K. (2013). Aberrant restoration of spines and their synapses in L-DOPA-induced dyskinesia: involvement of corticostriatal but not thalamostriatal synapses. J. Neurosci. 33, 11655-11667. doi: 10.1523/JNEUROSCI.0288-13.2013

Ztaou, S., Lhost, J., Watabe, I., Torromino, G., and Amalric, M. (2018). Striatal cholinergic interneurons regulate cognitive and affective dysfunction in partially dopamine-depleted mice. Eur. J. Neurosci. 48, 2988-3004. doi: 10.1111/ejn.14153

Ztaou, S., Maurice, N., Camon, J., Guiraudie-Capraz, G., Kerkerian-Le Goff, L., Beurrier, C., et al. (2016). Involvement of striatal cholinergic interneurons and M1 and M4 muscarinic receptors in motor symptoms of Parkinson's disease. J. Neurosci. 36, 9161-9172. doi: 10.1523/JNEUROSCI.0873-16.2016

Conflict of Interest Statement: The authors declare that the research was conducted in the absence of any commercial or financial relationships that could be construed as a potential conflict of interest.

Copyright (C) $2019 \mathrm{Du}$ and Graves. This is an open-access article distributed under the terms of the Creative Commons Attribution License (CC BY). The use, distribution or reproduction in other forums is permitted, provided the original author(s) and the copyright owner(s) are credited and that the original publication in this journal is cited, in accordance with accepted academic practice. No use, distribution or reproduction is permitted which does not comply with these terms. 